\title{
Role of Angiogenic Factors in Preeclampsia
}

Pradhan P

Department of Obstetrics and Gynaecology, Nepal Medical College Teaching Hospital, Kathmandu, Nepal.

Preeclampsia, the syndrome of hypertension, proteinuria, edema and hyperuricemia occurring during the last trimester of pregnancy remains one of the great mysteries. Recently gene expression profiling of placental tissue from healthy and preeclamptic women used to see which genes were up or down regulated in preeclamptic patients. Alterations in circulating angiogenic proteins correlated with disease severity, earlier onset of preeclampsia and birth of small for gestational age (SGA) fetus. These findings lend support to the hypothesis that circulating angiogenic proteins may have an important biological role in preeclampsia.

Keywords: angiogenic factors,eclampsia,preeclampsia.

Preeclampsia affects $5 \%$ of all pregnancies producing substantial maternal and perinatal morbidity and mortality. ${ }^{1}$ Although our understanding of preeclampsia has increased over the past 50 years, the etiology is still incomplete and the management remains supportive: close observation, treatment with antihypertensive agents and magnesium sulphate and delivery of the fetus. It occurs only in the presence of placenta even when there is no fetus and remits dramatically postpartum., ${ }^{2,3}$ Placenta from severe preeclampsia classically have numerous infarcts, sclerotic narrowing of arteries and arterioles and fibrin deposition and thrombosis. The characteristic placental lesion in severe preeclampsia is due to diminished endovascular invasion by cytotrophoblast and failure of uterine spiral arteriolar remodeling. ${ }^{4}$ Based on this observation, it has been suggested that there may be a circulating factors of placental origin which affect systemic endothelial cell function and lead to preeclampsia. Many candidate factors including tumor necrosis factor (TNF-alpha), Interleukin-6, IL-1a, IL-1b, neurokinin B, asymmetric dimethyl L-arginine (ADMA) have been suggested, none so far have been proven to be etiologic. ${ }^{5-7}$

Recently gene expression profiling of placental tissue from healthy and preeclamptic women used to see which genes were up or down regulated in preeclamptic patients. One consistently up-regulated

\section{CORRESPONDENCE}

Dr Pramila Pradhan

Department of Obstetrics and Gynaecology

Nepal Medical College Teaching Hospital, Kathmandu, Nepal

Email:drpramilapradhan@gmail.com

Phone: +977-9841490496 protein turned out to be a soluble fms-like Tyrosine -kinase-1 (sFlt-1). ${ }^{8}$ This protein acts by adhering to the receptor-binding domains of placental growth factor (PIGF) and vascular endothelial growth factor (VEGF) the angiogenic factors, preventing their interaction with endothelial receptors on the cell surface and thereby inducing endothelial dysfunction. In vivo study, sFlt-1 was administered to pregnant and non-pregnant rats and it produced hypertension, proteinuria and glomeruloendotheliosis that mimics the human syndrome of preeclampsia demonstrating for the first time a clear cause and effect relationship between this protein and this disease. ${ }^{8}$ In another in vivo study, it was observed that there is a marked rise in circulating sFlt- 1 concentration beginning about 5-6 weeks before the onset of clinical preeclampsia accompanied by decreases in the circulating free PIGF and VEGF. ${ }^{9}$ The loss of these growth factors damage the maternal small blood vessels producing diverse symptoms of preeclampsia and eclampsia. Moreover alterations in these circulating angiogenic proteins correlated with disease severity, earlier onset of preeclampsia and birth of small for gestational age (SGA) fetus. These findings lend support to the hypothesis that circulating angiogenic proteins may have an important biological role in preeclampsia. ${ }^{8,9}$ In vitro evidence as described by Maynard showed higher sFlt-1 concentration in the blood of preeclamptic versus healthy pregnant women which dropped to normal within 48 hours of birth. ${ }^{8}$ Merchan in a vitro angiogenetic assay showed that blood from preeclamptic women blocked the vessels growth and the treatment with exogenous VEGF or PIGF reversed the antiangiogenic properties of preeclamptic serum. ${ }^{8}$ The inhibiting effect of serum from women with 
preeclampsia disappeared after delivery, suggesting that the factor may be released by the placenta. Thus the antiangiogenic effects of sFlt-1 may account for many of the manifestations of preeclampsia including the unique glomerular effects. There is evidence from animal model that VEGF is important in maintaining glomerular endothelial cell health and healing. ${ }^{9}$ In antiangiogenic oncology trial, antagonism of VEGF using neutralising antibodies and VEGF receptor inhibitor can produce headache, hypertension, proteinuria and coagulopathy in human subjects..$^{10,11}$ Therefore by neutralising VEGF and PIGF, excess sFlt-1 may have a contributory role in the pathogenesis of the maternal syndrome of preeclampsia. The hypothesis that excessive production of sFlt-1 may play a causal role in the preeclampsia is supported by recent studies that reported a link between trisomy 13 pregnancies and circulating angiogenic protein concentrations during the first and second trimester. Fetuses with an extra copy of this chromosome should theoretically produce more of these gene products than their normal counterparts. ${ }^{12}$ The ratio of circulating sFlt-1 to PIGF was recently shown to be significantly increased in these women thus accounting for the increased risk of preeclampsia. ${ }^{13}$

Endoglin a co-receptor for transforming growth factor $\mathrm{B}_{1}$ and $\mathrm{B}_{2}\left(\mathrm{TGFB}_{1} / \mathrm{TGFB}_{2}\right)$ is highly expressed on the cell membrane of vascular endothelium and syncytotrophoblast. ${ }^{14}$ Placental endoglin is up-regulated in preeclampsia, releasing soluble endoglin into maternal circulation. Soluble endoglin is an antiangiogenic protein that may inhibit TGF-B, signaling in vasculature Adenoviral mediated over expression of both sFlt-1 and soluble endoglin caused severe vascular damage, nephrotic range proteinuria, severe hypertension, a syndrome similar to the HELLP syndrome. ${ }^{15}$ Along with experimental evidence in rodents, these data suggest that circulating soluble endoglin and sFlt-1, each of which causes endothelial dysfunction by a different mechanism, may both contribute to the syndrome of preeclampsia..$^{14,15} \mathrm{How}$ placental dysfunction is related to placental sFlt-1 production and why placental perfusion is deranged in preeclampsia remains unknown. Recent data of in vitro primary cytotrophoblast cultures suggest that placental hypoxia may play an important role in up regulating sFlt-1 production and this up regulation of sFlt-1 may be through genetic, environmental or immunological.
The next step is, will measurement of blood sFlt-1, VEGF and PIGF levels allow us to develop a test that can predict the development of preeclampsia before the onset of symptoms. Few studies have concentrated on examining the potential ability of sFlt-1 and PIGF level as biomarkers in the diagnosis and prediction of preeclampsia. Most of the studies have looked statistically at sFlt-1 as a potential predictor of preeclampsia. ${ }^{16}$ Examining odd ratios, sensitivity and specificity for various sFlt-1 cut off values in different trimesters has yielded the conclusion that higher the sFlt-1 levels the more predictive it is of preeclampsia. PIGF a smaller protein is decreased in the urine of women with preeclampsia compared with normal pregnancy. Similar to serum PIGF, urinary levels were lowest in the preeclampsia group with active disease regardless of gestational age at the time of onset of symptoms. It was concluded that urinary PIGF concentration during mid pregnancy was low only in the setting of preeclampsia. When urinary PIGF was combined with a serum sFlt-1 and PIGF ratio (ratio $>10$ suggesting preeclampsia), all of the cases destined to develop preeclampsia within the following 5 weeks could be distinguished from the control pregnant women. ${ }^{17}$ Thus a two-step approach of initial urinary screening of PIGF followed by serum sFlt-1/PIGF in the women who have low urinary PIGF levels may be a cost-effective approach for the screening of preeclampsia. If a reliable and valid urinary dipstick assay can be developed, one scenario might be to screen all women for detection of low urinary PIGF concentration. Among those with low levels, serial serum measurements of sFlt1 and PIGF could then be used to identify more precisely the individual at high risk of developing pre-eclampsia. Prospective longitudinal studies with measurements throughout pregnancy are needed to assess the validity of observations.

\section{DISCLOSURE}

The authors report no conflicts of interest in this work.

No violation of human rights and safety.

Funding: Nil 


\section{REFERENCES}

1. Walker JJ. Preeclampsia. Lancet. 2000; 356:1260-5 .

2. Robert JM, cooper Dw. Pathgenesis and genetics of pre eclampsia. Lancet. 2001; 357:53-6.

3. Page EW. The relation between hydatidiform mole, relative ishcaemia of the gravid uterus and the placental origin of eclampsia. Am J Obstet Gyanecol. 1939;37:2913 .

4. Robert JM, Taylor RN, Musci TJ, Rogers GM, Hubel CA, Mclaughin MK, et al. Preeclampsia: an endothelial cell disorder. Am J Obstet Gyanecol. 1989;1611:1200-4.

5. Robert JM. Preeclampsia: what we know, what we don't know. Semin Perinatol 2000;24:24-8.

6. Page NM, Wood RJ, Gardinor SM, Lomthaisong K, Gladwell RT, Butin DJ, et al. Excessive placental secretion of neurokinnin during third trimester causes preeclampsia Nature. 2000;405:797-800.

7. Avvidu MD, Hingorani AD, Tsikas D, Frolich JC, Vallance $\mathrm{P}$, Nicolaides $\mathrm{KH}$, et al. Endothelial dysfunction and raised plasma concentration of asymmetric dinethyl-1 arginine in pregnant women who subsequently develop preeclampsia. Lancet 2003;361:1511-7.

8. Mynard SE, Min JY, Merchant J, et al. Excess placental soluble fms-like tyrosine kinase-1 (sFlt-1) may contribute to endothelial dysfunction, hypertension and proteinuria in pregnancy. J Clin Invest. 2003;111:649-58.

9. Levine RHJ, Maynard SE, Quan C, Lim KH, England LJ, $\mathrm{Yu} \mathrm{KF}$, et al. Circulating angiogenic factors and the risk of preeclampsia. N Eng J Med. 2004;350:672-83.

10. Kabbinavar F, Hernitz HI, Fehrenbacher L, Meropol NJ, Novotiny WF, Lieberman G, et al. Phase II randomized trail comparing Bivalizymab plus Florouracil (Fu), Leucovorin (Lv) with $\mathrm{Fu} / \mathrm{Lv}$ alone in patients with metastatic colorectal cancer. J Clin Oncol. 2003;21:60-5.

11. Yang JC, Haworth L, Sherry RM, Hwu P, Schwartzentruber DJ, Topalian SL, et al. A randomised trial of Bevacizymab an antivascular endothelial growth-factor antibody for metastatic renal cancer. N Eng J Med. 2003;349:427-34

12. Chaiworanponsa T, Romero R, Espinoza J, Busold E, Kim YM.. Evidence supporting a role for blockade of the vascular endothelial growth factor system in the pathophysiology of preeclampsia. Am J Obstet Gyanecol. 2004;190:1541-50.

13. Masuda Y, Shinifu A, Mori J, Shimizu A, Ishiwata T, Kitamura $\mathrm{H}$, et al Vascular endothelial growth factor enhanced glomerular capillary repair and accelerated resolution of experimentally induced glomerulonephrits. AM J Pathol. 2001;159;599-608.

14. Levine RJ, Lam C, Qina C, Maynard SE, Yu KF, Sachs BP, Sibia BM. Soluble endoglin and other circulating anti angiogenic factors in preeclampsia. N Eng J Med. 2006;355:992-1005.

15. Venkatesha S, Toporsian M, Lam C, Hanai J, Mammoto T, Kim YM. Slouble endogloin contributes to the pathogenesis of preeclampsia. Nat Med 2006;12:642-9.

16. Chaun L, $\lim$ K H, Karumachi SA. Circulating angiogenic factor in the pathogensis and prediction of pre eclampsia. Hypertension. 2005;49;1077-85.
17. Levin RJ,. Thandani R, Qian C, Lam C, Lim KH, YU $\mathrm{KF}$, et al. Urinary placental growth factor and risk of preeclampsia. JAMA 2005;293(I):77-85. 